\title{
Mask on the Emotions: the Role of Emotional Labor on Mental Health and Resiliency of Nurses
}

\author{
Manijeh Firoozi $^{1, *}$, id, Hammed Shakouri ${ }^{2}$
}

\author{
${ }^{1}$ Assistant Professor, Department of Psychology, Faculty of Psychology and Education Science, University of \\ Tehran, Iran \\ ${ }^{2}$ MSc, Department of Management, University of Tehran, Iran \\ * Corresponding author: Manijeh Firoozi, Assistant Professor, Department of Psychology, Faculty of \\ Psychology and Education Science, University of Tehran, Iran. E-mail: mfiroozy@ut.ac.ir
}

Received: 24 Apr 2018

Accepted: 05 Jan 2018

Abstract
Introduction: Emotional labor tend to consider manage and expression feeling at workplace. The main
purpose of this study was investigate role of emotional labour and resiliency in mental health of nurses.
Methods: This study used a descriptive disign. According to the Morgan table, 312 nurses from Qamar Bani
Hashem Hospital and Shaheed Madani Hospital in Khoy were participated in this study. They completed
three questionnaires including Zapf Emotional Labor scale, General Health Questionnaire (GHQ) and
Connor and Davidson Resilience Scale. Finally, the data were analyzed through stepwise regression analysis.
Results: Surface emotional acting had a positive and significant relationship with physical symptoms, anxiety
and insomnia, social dysfunction and depression (P < 0.001$)$, while its relationship with resilience and work
experience was negatively significant (P < 0.001$)$. Meanwhile, Surface emotional acting explain to $24 \%$ of
mental health in nurses (negatively). Deep emotional acting had a meaningful and positive relationship with
the components of mental health, resilience and work experience. In other words, with growth work
experience, tend to increase deep emotional acting. In the result, resilience and mental health improve
gradually.
Conclusions: Surface emotional acting, often seen in little background nurses, could lead to cognitive
dissonance and inconsistency between behavior and beliefs. In the result, it can reduce psychological health.
If use consistently defensive mechanisms to control these emotional disruptions, the nurses will be experince
burnout in long time.
Keywords: Expressed Emotion, Psychological Resilience, Mental Health, Nurse, Occupational Health (c) 2018 Iranian Nursing Scientific Association (INA) 


\title{
نقابى بر عواطف: نقش بازيكرى احساسى بر سلامت روان شناختى و تاب آورى يرستاران
}

\author{
منيزه فيروزى ا.*3id) حامد شكورى '
}

' استاديار، كروه روانشناسى، دانشكده روانشناسى و علوم تربيتى، دانشكاه تهران، تهران، ايران

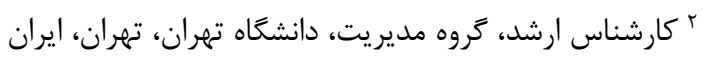

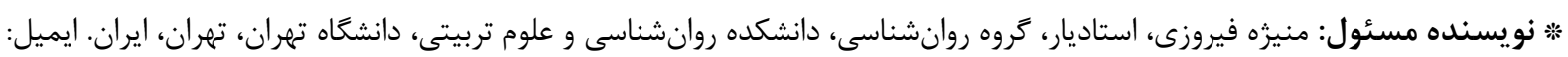
mfiroozy@ut.ac.ir

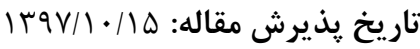

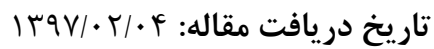

مقدمه: بازيخرى احساسى به مديريت و نحوه بيان احساسات و هيجانها در محيط كار اشاره دارد. هدف اصلى اين مطالعه نقش بازيكرى

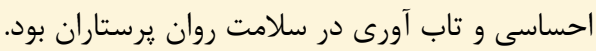

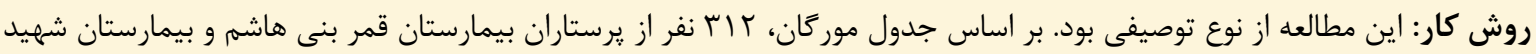

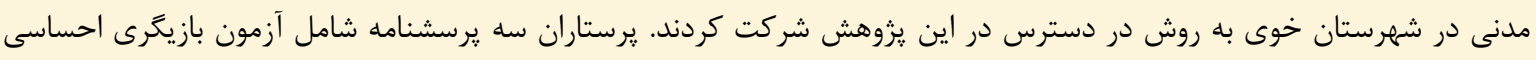

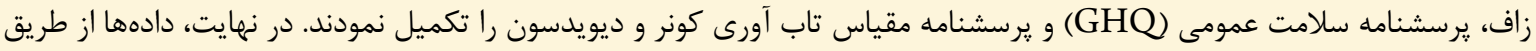
تحليل ركرسيون كام به كام تحليل شدند. برافي

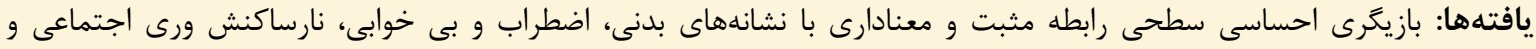

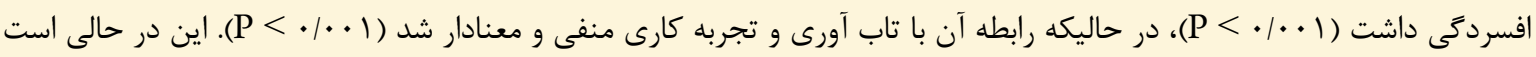

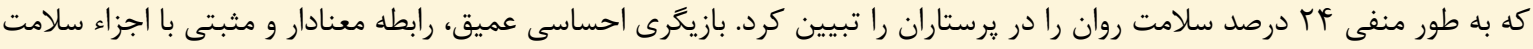

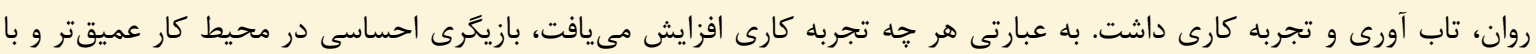

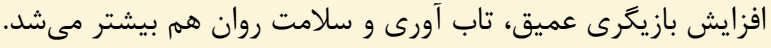

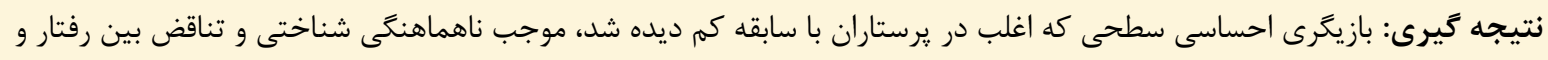

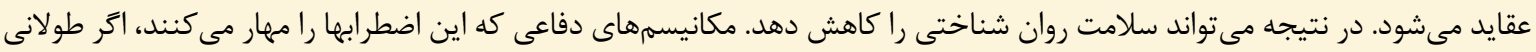

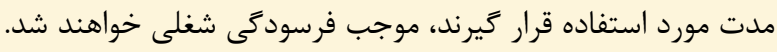

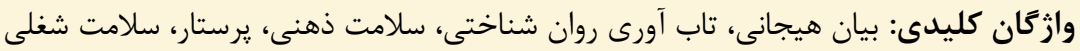

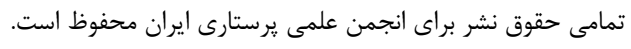

است. گوسيرند و ديفندرف [؟] بازيخرى احساسى را رفتارهاى بركرفته

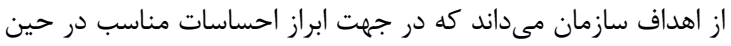

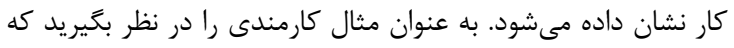
مسئول رسيدكى به شكايات مشتريان است و هر روز بايد به به شكايات

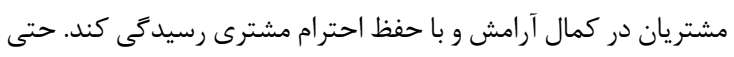

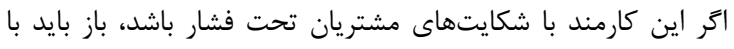

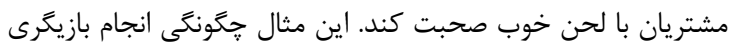

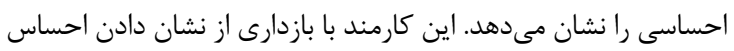

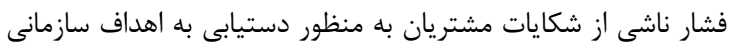

مقدمه بازيخرى احساسى در محيطهاى شغلى اجتناب نايذير است و افراد در

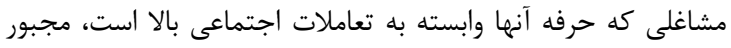

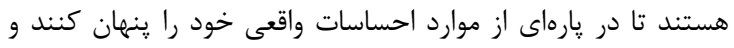

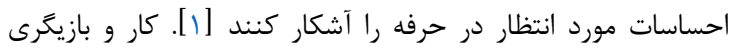

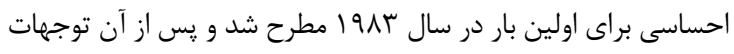

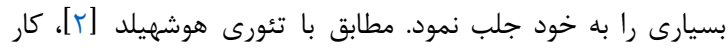
احساسى نوعى تنظيم احساسات شخص است تا فرد برخى از احساسات

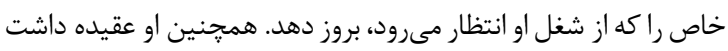

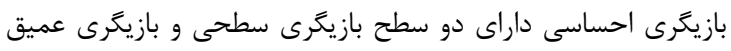




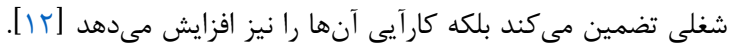

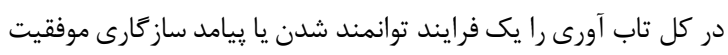

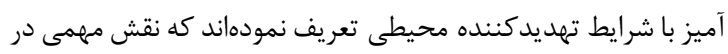

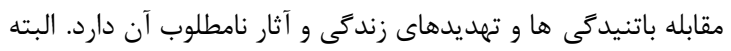

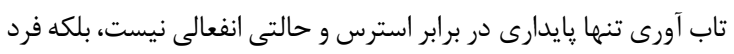

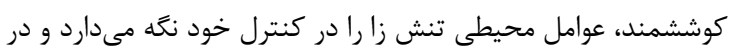

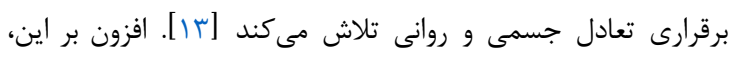

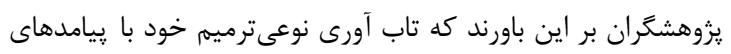

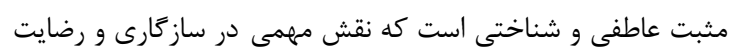

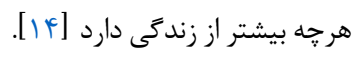

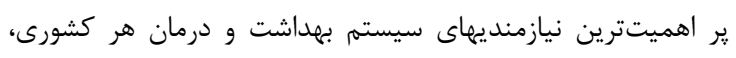

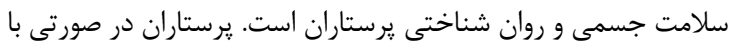

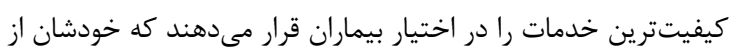

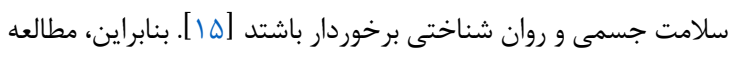

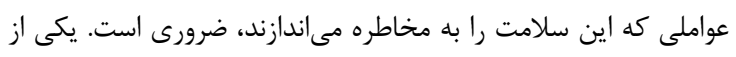

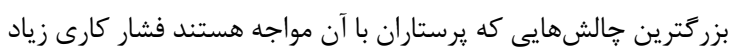

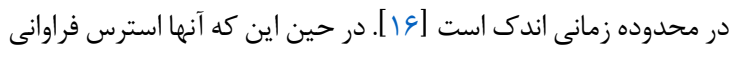

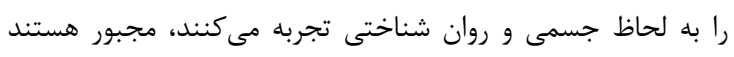

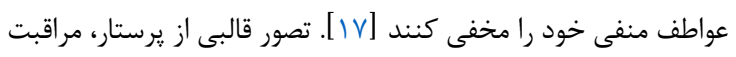

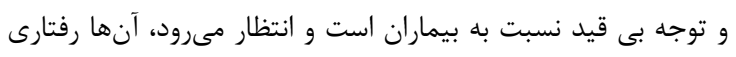

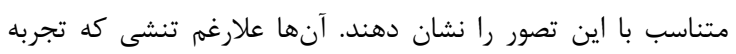

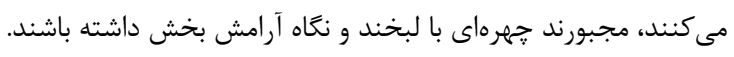

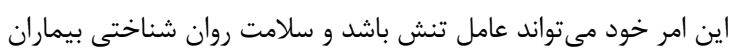

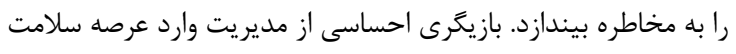

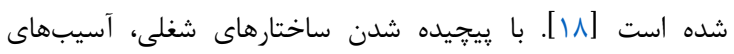

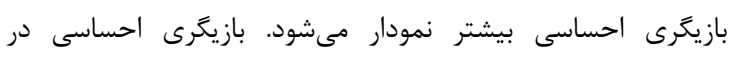

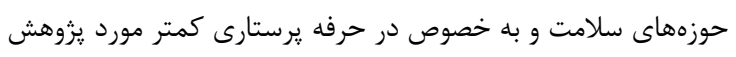

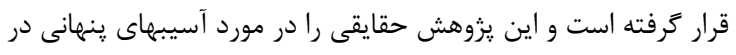

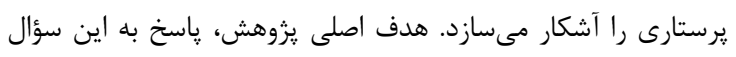

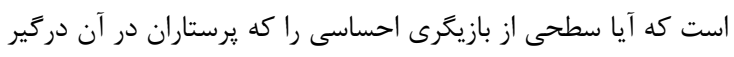

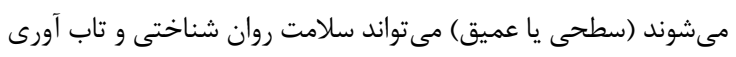
آنها بيش بينى كند.

\section{روش كار}

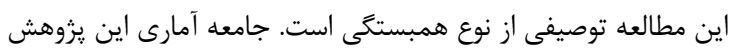

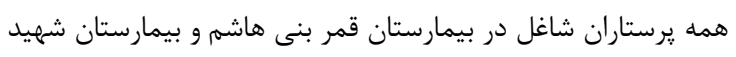

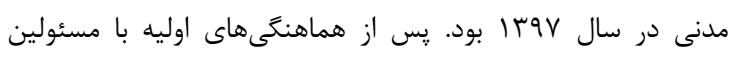

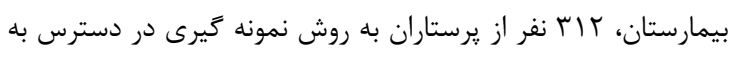

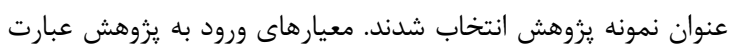

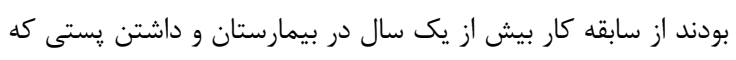

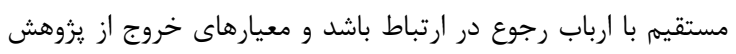

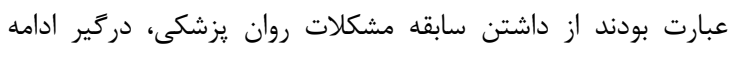

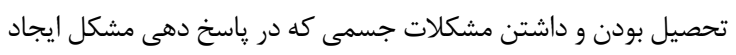

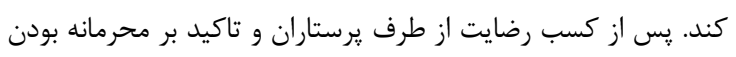

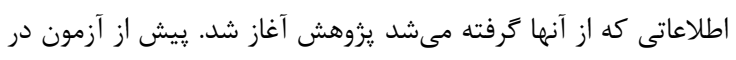

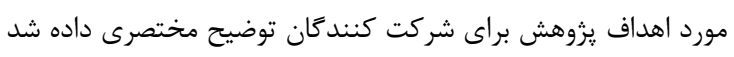

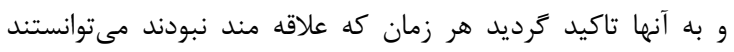

مورد انتظار، همجنان با آرامى و متانت با مشتر يان برخورد مى كند [؟] ئ.

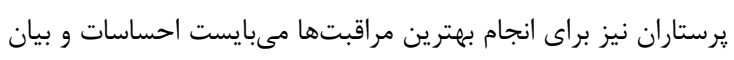

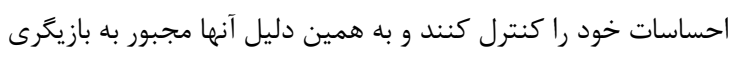

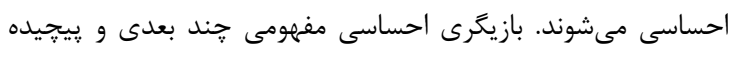

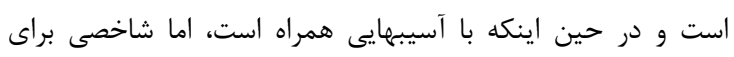

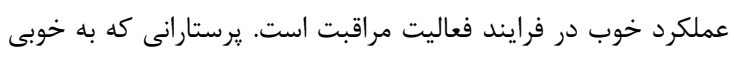

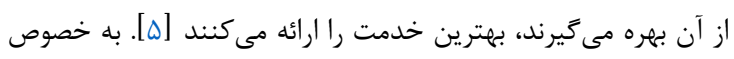

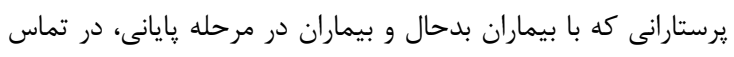

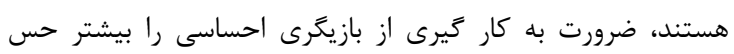

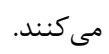

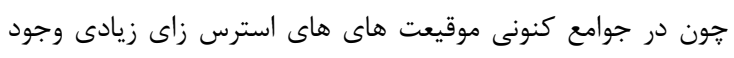

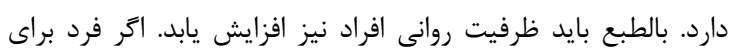

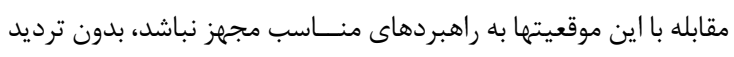

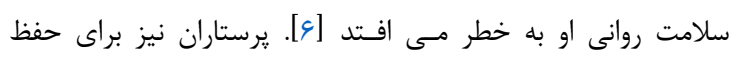

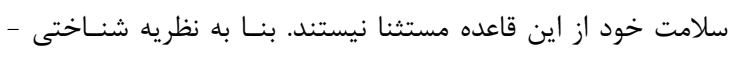

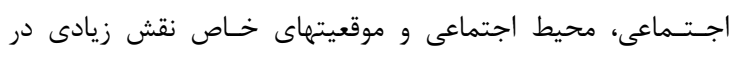

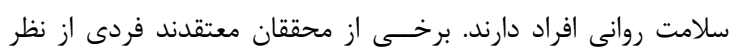

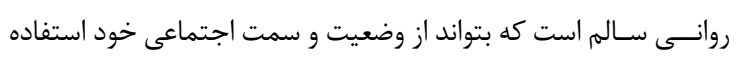

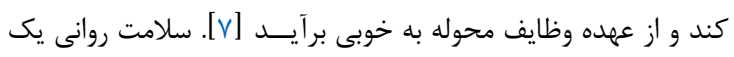

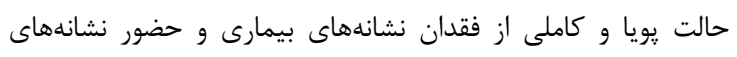

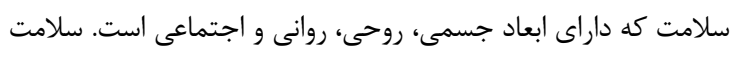

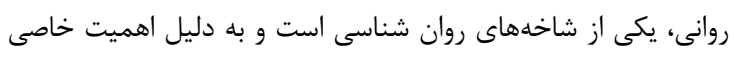

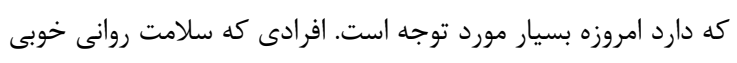

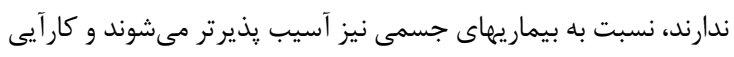

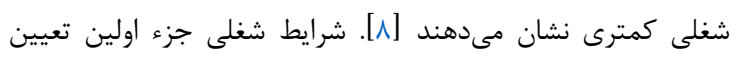

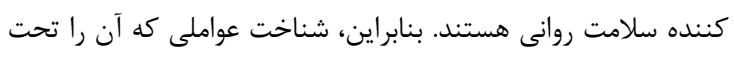

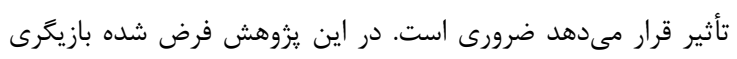

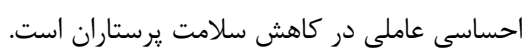

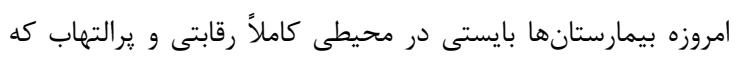

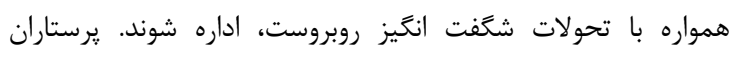

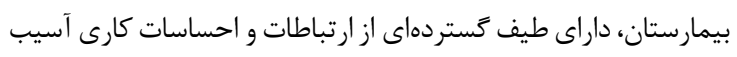

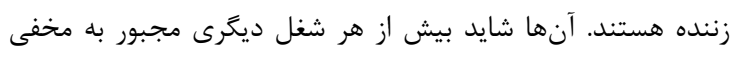

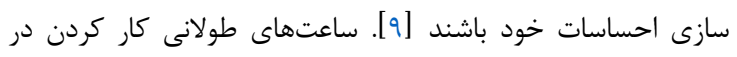

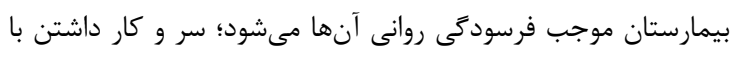

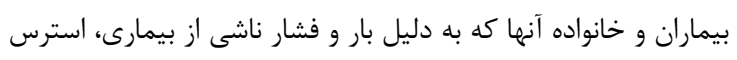

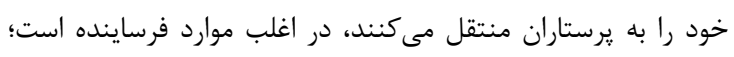

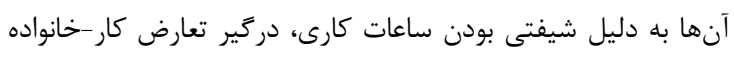

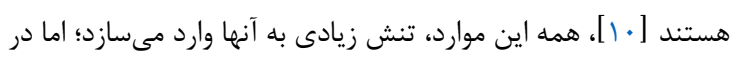

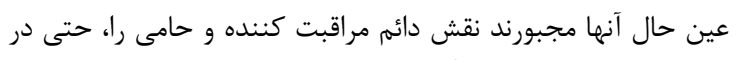

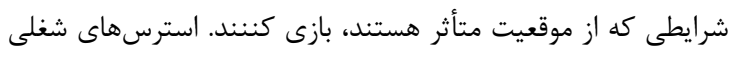

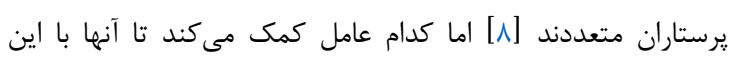

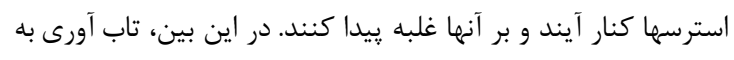

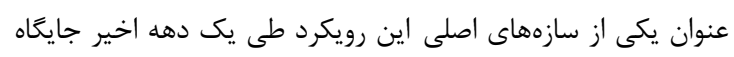

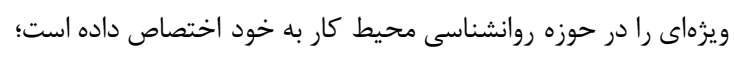

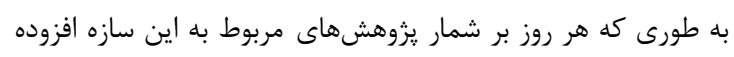

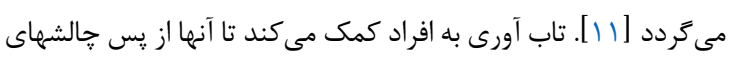

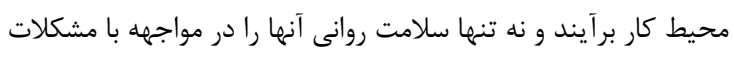


محمدى و همكاران، براى تعيين روايى در محاسبه همبستخى هر نمره

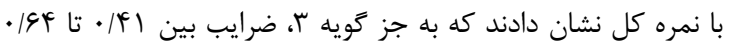

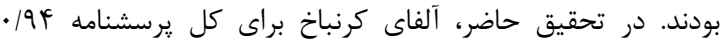
محاسبه شد كه بيانگر ضريب پايايى مطلوب است.

يافتهها

در اين يزوهش ميانگين سن شركت كنندكان هـ سال و انحراف معيار

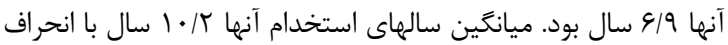

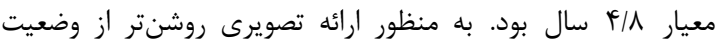

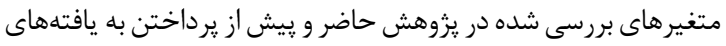

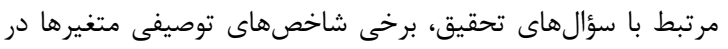
جدول ا ارائه شده است.

جدول ا: ميانكين و انحراف معيار متغيرهاى بازيخرى احسلسى، سلامت روان و تاب

\begin{tabular}{|c|c|c|}
\hline انحراف از معيار & ميانغين & متغيرها \\
\hline $\mid r / r r$ & $r \cdot / v 9$ & بازيخرى احساسى عميق \\
\hline $1.14 \Delta$ & FF/TA & بازيگرى احساسى سطحى \\
\hline$\Delta / \Delta \varphi$ & $\mid r / \Delta F$ & نشانه هاى بدنى \\
\hline$r / \Delta V$ & $10 / 9 V$ & اضطراب و بى خوابى \\
\hline $914^{4}$ & $14 / \pi q$ & نارساكنشورى اجتماعى \\
\hline$F / \& \Delta$ & $I V / r \Lambda$ & افسردكى وخيم \\
\hline $9 / 9$ & $r \Delta / 9 \Lambda$ & سلامت روان (نمره كل) \\
\hline$V / T$ & $r q / \pi r$ & تاب آورى \\
\hline
\end{tabular}

نتايج آزمون همبستكى بِيرسون نشان داد كه بين حوزههاى بازيخرى

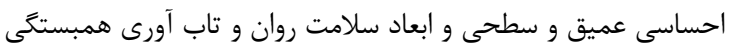

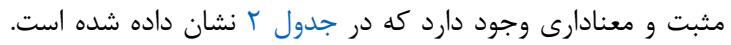

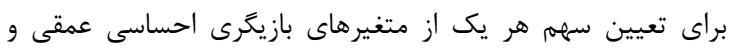

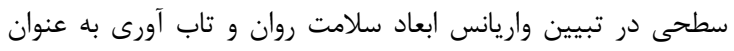

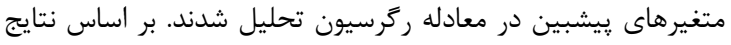
جدول r مشاهده شده معنادار است.

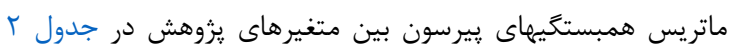

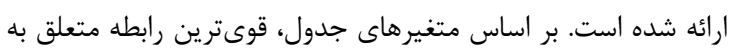

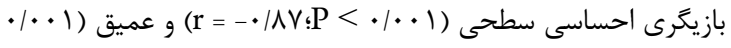

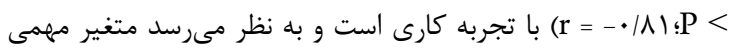

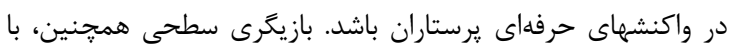

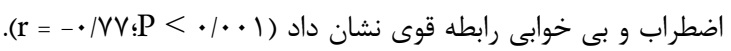

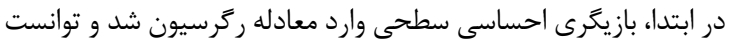

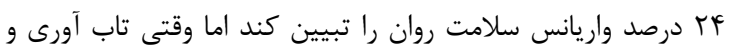

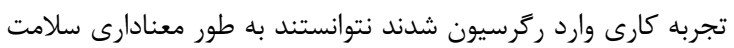

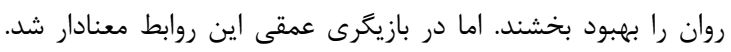

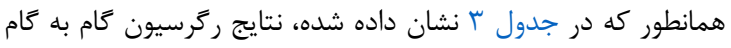

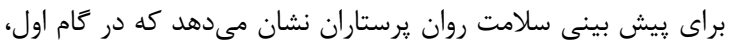

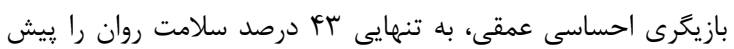
بينى مى كند. در كام دوم، بازيكرى احساسى سطحى به همراه تاب آورى 9 ه درصد

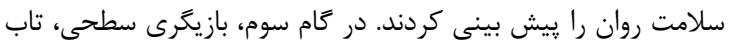

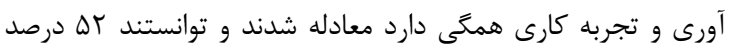

يروهش را ترك كنند. 9 نفر در نهايت به دليل عدم علاقه مندى، در

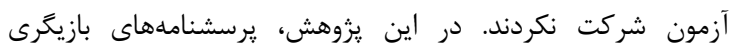

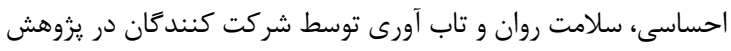

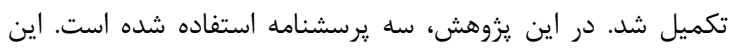

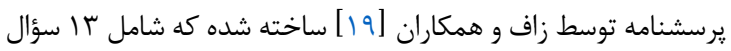

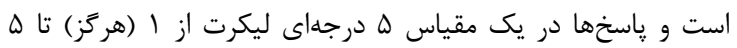

(هميشه) قرار مى آنيرند.

اين يرسشنامه بازيخرى احساسى سطحى و و عميق رانى را از هم متمايز

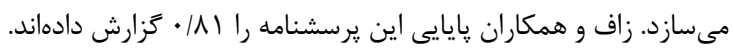

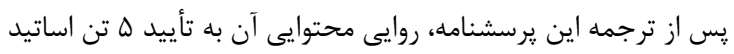

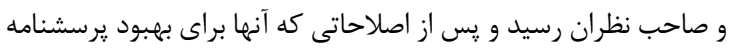

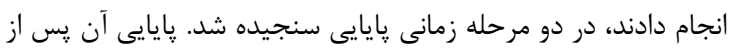

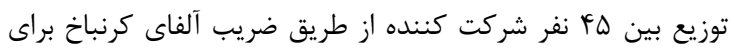

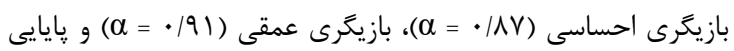

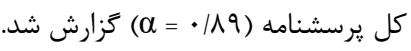

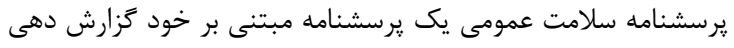

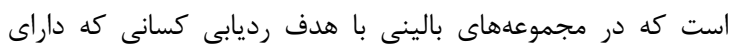

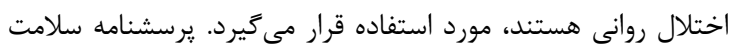
عمومى (GHQ) توسط منتظرى و همكاران [•r] تنظيمى و به منظور

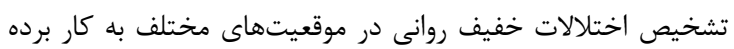

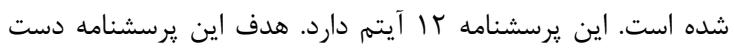

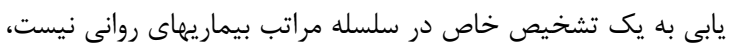

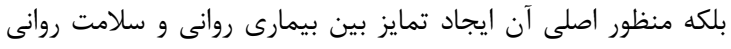

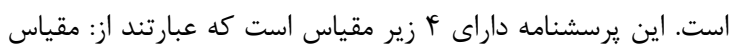

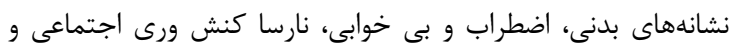

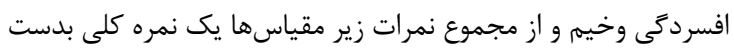

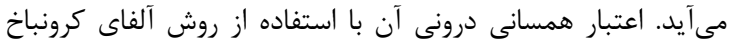

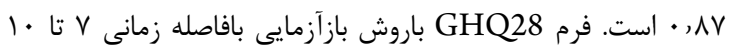

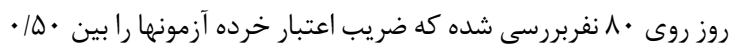

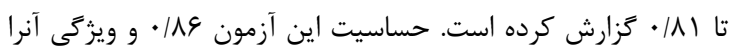

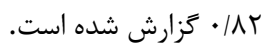

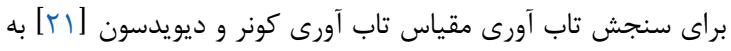

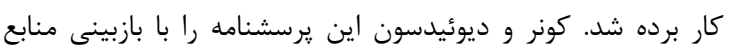

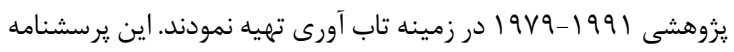

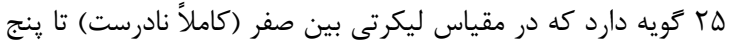

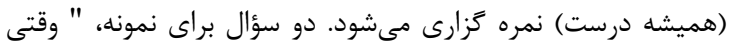

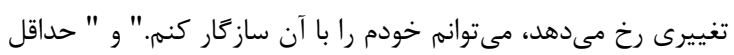

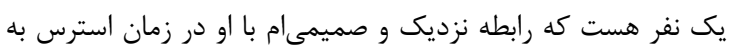
من كمك كند."

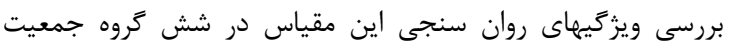

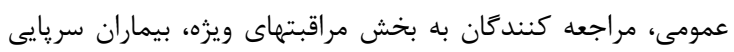

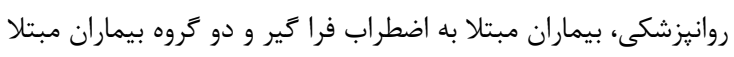

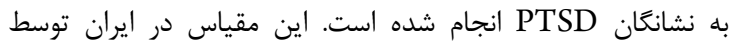

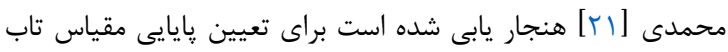

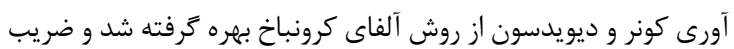

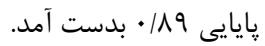


سطحى، تاب آورى و تجربه كارى بيش بينى كننده سلامت روان

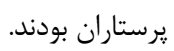

سلامت روان يرستاران را بيش بينى كنند. بر اساس يافتهها بازيكرى

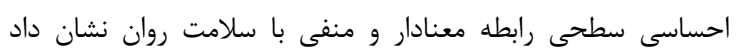

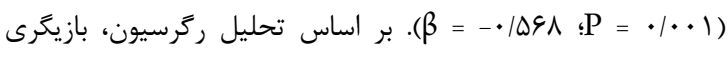

\begin{tabular}{|c|c|c|c|c|c|c|c|c|}
\hline \multirow[t]{8}{*}{$\wedge$} & v & 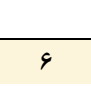 & $\Delta$ & $f$ & $r$ & تاب آورى & سى، سلامت & متغول rا: ماتريس همبستخى بين مـ \\
\hline & & & & & & & 1 & 1- بازيكرى احساسى سطحى \\
\hline & & & & & & 1 & $\cdot 199^{* * *}$ & ז-بازيگرى احساسى عميق \\
\hline & & & & & 1 & $-\cdot \mid \Delta \Lambda^{* \frac{* \omega}{*}}$ & $\cdot|9|^{* * *}$ & r-نشانههاى بدنى \\
\hline & & & & 1 & $\left.\cdot 19\right|^{* \ldots}$ & $-\cdot 109^{* \%}$ & $\cdot / V V^{* a}$ & f-اضطراب و بى خوابى \\
\hline & & & 1 & $\cdot /\left.V\right|^{m: *}$ & $\cdot|f| *$ & $-\cdot|9|^{* * * a}$ & $\cdot|9|^{* * a}$ & ه-نارساكنشورى اجتماعى \\
\hline & & 1 & $\cdot|\Delta|^{* \#}$ & $\cdot 199^{* 0}$ & $\cdot / V \varphi^{* * *}$ & $\cdot / r \Lambda$ & $\cdot \mid 9 Y^{H_{*}^{*}}$ & צ-افسردگى \\
\hline & 1 & $-\cdot / \mathbb{A} \Delta^{*}$ & $-\cdot / r \Lambda$ & $-\cdot / 49^{m *}$ & $-\cdot|4|^{*}$ & $\cdot 19 \mathrm{~V}^{* * * *}$ & $-\cdot 19 r^{* * *}$ & V-تاب آورى V \\
\hline 1 & $\cdot 19 \Lambda^{* m}$ & $\cdot / 4 t$ & $-\cdot / \mathcal{A} r^{*}$ & $\cdot|\Delta|^{* * *}$ & $\cdot / 4 r^{*}$ & $\cdot|\Lambda|^{* a}$ & $-\cdot / \Lambda V^{* * m}$ & ^-تجربه كارى (به سال) \\
\hline
\end{tabular}

جدول זّ: خلاصه مدل ركرسيون، تحليل واريانس و مشخصههاى آمارى ركرسيون كام به كام بازيخرى احساسى عميق و تاب آورى بر سلامت روان يرستاران

\begin{tabular}{|c|c|c|c|c|c|c|}
\hline $\mathbf{P}$ & $\mathbf{R}^{2}$ & F & $\beta$ & SE B & B & تتغير \\
\hline & & & & & & \\
\hline \multirow[t]{2}{*}{$\cdot 1 \cdots 1$} & TKT & $\mid A V / F \varepsilon \Delta$ & $\cdot / \Delta \& \Lambda$ & 每 & $\cdot|r| F$ & بازيكرى عميق \\
\hline & & & & & & 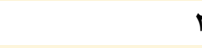 \\
\hline$\cdot \mid \cdots 1$ &.$/ 4 \wedge 9$ & $111 / 99 \mathrm{~V}$ & $\cdot / \& \Delta \Delta$ & $\cdot \pi 4$ & . /rrF & بازيخرى عميق \\
\hline \multirow[t]{2}{*}{$\cdot 1 \cdots 1$} & $\cdot / 4 \wedge 9$ & $111 / 98 \mathrm{~V}$ & $\cdot / T V R$ & .1 .94 &.$/ 199$ & تاب آورى \\
\hline & & & & & & r \\
\hline$\cdot 1 \cdots 1$ & $\cdot|\Delta T|$ & $9 N / \cdot 4 T$ & $V N / \cdot r I$ & . & $\cdot / r \cdot r$ & بازيكرى عميق \\
\hline$\cdot 1 \cdots 1$ & $\cdot|\Delta T|$ & $Q N / F T$ & $V N / \cdot r I$ & .1 .99 & . KIV & تاب آورى \\
\hline $.1, \ldots 1$ & $\cdot|\Delta T|$ & QN/FT & $V N / \cdot r I$ & $.1+\% \Delta$ & .1119 & تجربه كارى \\
\hline
\end{tabular}

هيجانى تعديل مىشود [rV] نتايج يزوهش حاضر، بر حسب احتمالات زير تبيين مى كردند:

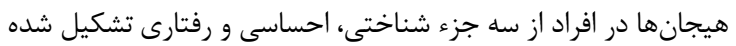

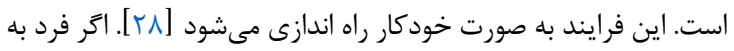

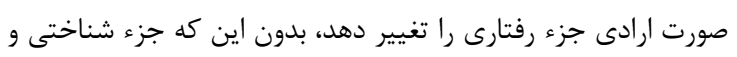

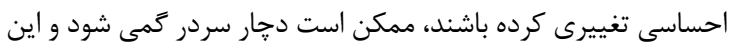

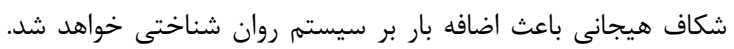

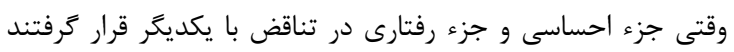

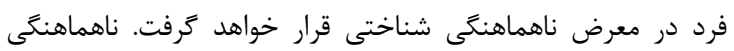

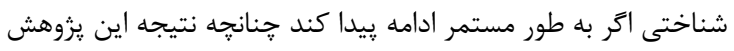

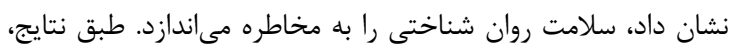

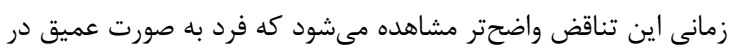
بازيخرى خود فرو رفته باشد.

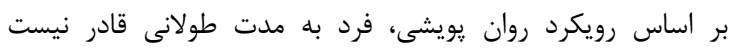

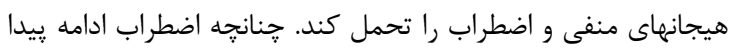

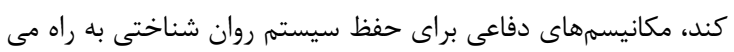

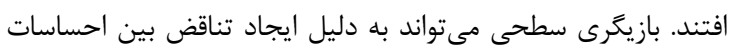

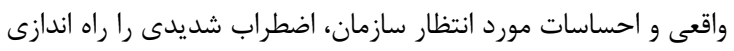

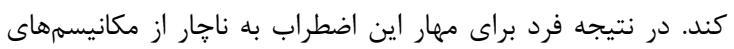

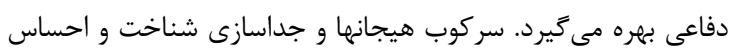

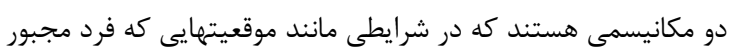

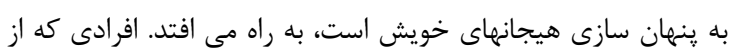

بر اساس يافتهاى يزوهش حاضر، بازيخرى احساسى سطحى رابطه

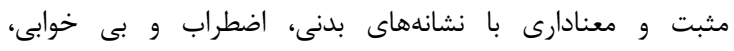

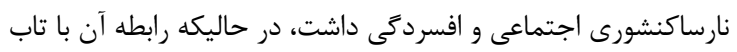

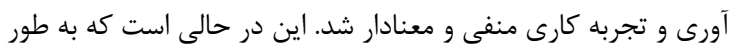

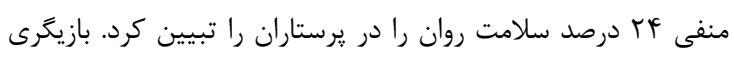

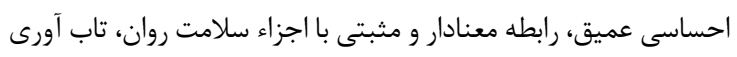

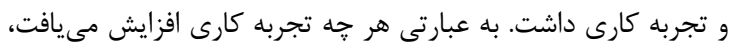

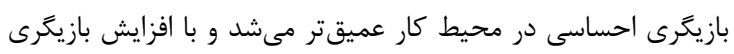

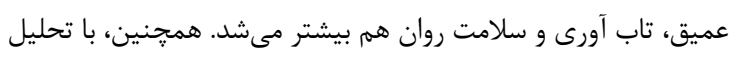

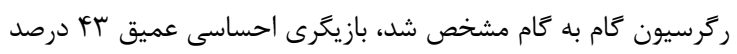

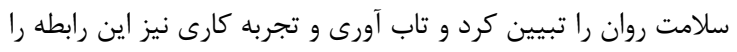

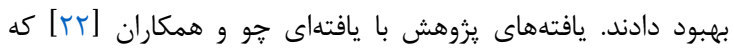
بازيغرى احساسى را در محيطهاى خرده فروشى نشان دادئه دادند، با يافته

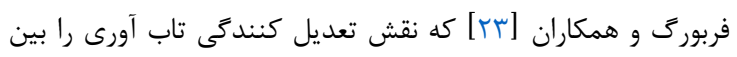

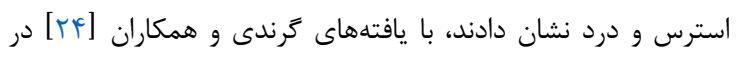

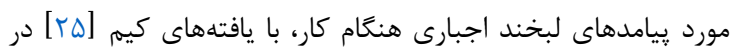

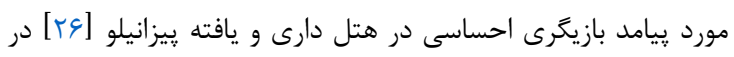
مورد اثر بازيكرى و شغل احساسى در سلامت شغلى همخوانى دارئ دارد.

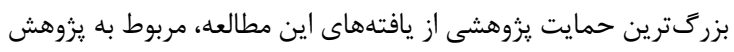

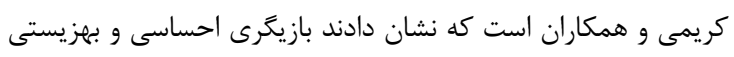

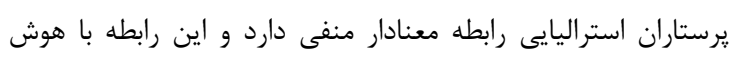


بازگشت به تعادل اول يا رسيدن به تعادل سطح بالاتر دارد و از اين رو

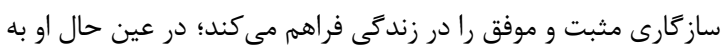

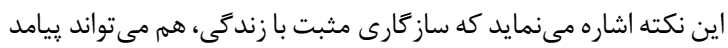

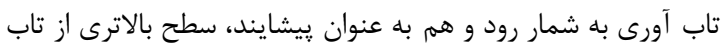

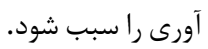

\section{نتيجه تيرى}

عوامل بسيارى وجود دارد كه رابطه بين بازيخرى احساسى و سلامت

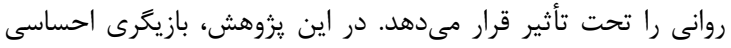

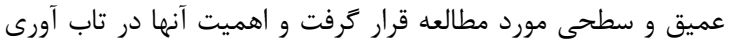

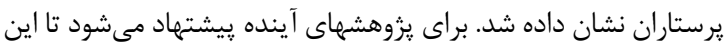

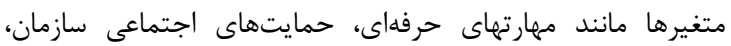

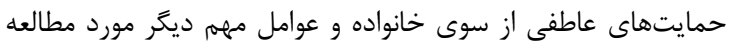

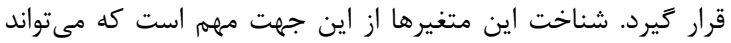

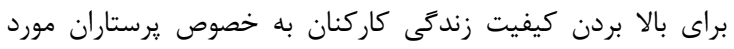

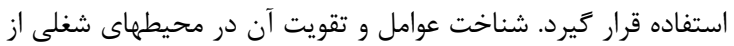

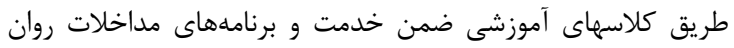

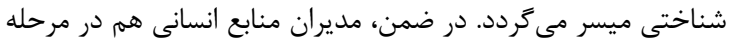

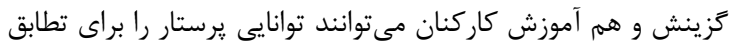

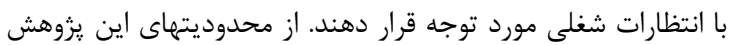

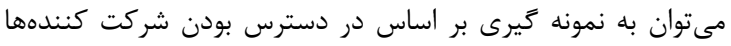

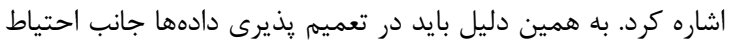

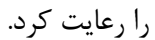
سياسگزارى از كليه مسئولين بيمارستان قمر بنى هاشم و بيمارستان شهيد مدنى

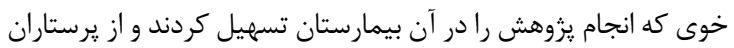

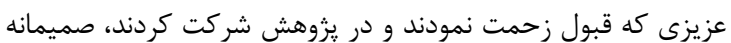
تشكر مى كنيه. تضاد منافع نويسندًان مقاله اعلام مىدارند كه در نعارش اين مقاله هيج كَونه تضاد

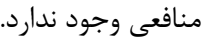

\section{References}

1. Lovatt M, Nanton V, Roberts J, Ingleton C, Noble B, Pitt E, et al. The provision of emotional labour by health care assistants caring for dying cancer patients in the community: a qualitative study into the experiences of health care assistants and bereaved family carers. Int J Nurs Stud. 2015;52(1):271-9. doi: 10.1016/j.ijnurstu.2014.10.013 pmid: 25468132

2. Mauno S, Ruokolainen M, Kinnunen U, De Bloom J. Emotional labour and work engagement among nurses: examining perceived compassion, leadership and work ethic as stress buffers. J Adv Nurs. 2016;72(5):1169-81. doi: 10.1111 /jan. 12906 pmid: 26841277

3. Lumsden K, Black A. Austerity policing, emotional labour and the boundaries of police work: an ethnography of a police force control room in England. Br J Criminol 2017;58(3):606-23.
بازيخرى سطحى استفاده مى كردند (بين رفتار و احساساتشان تناقض

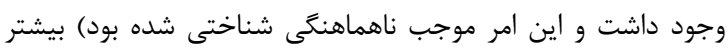

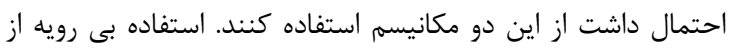

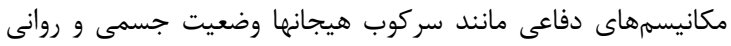

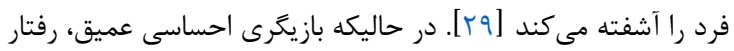

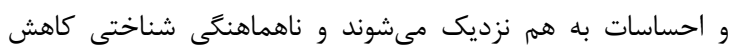

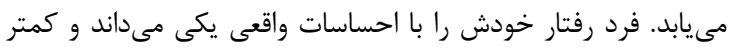

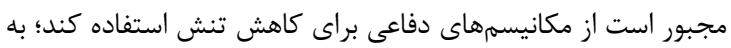

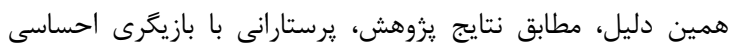

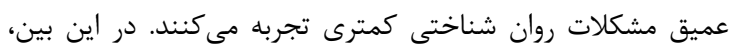

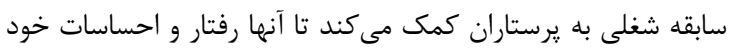

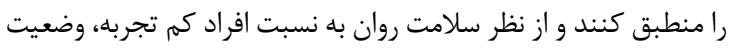
بهترى داشته باشند. نتيجه ديخر بزوهش اين بود كه در رابطه بين بازيخرى احساسى عميق

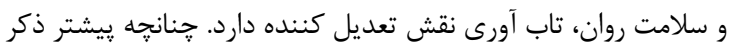

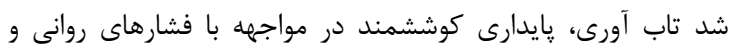

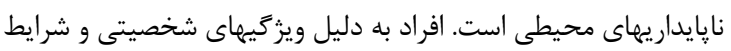

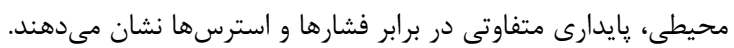

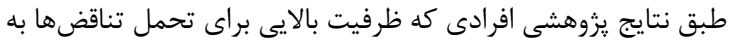

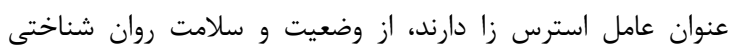

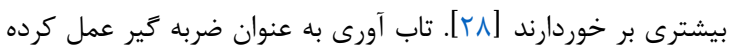

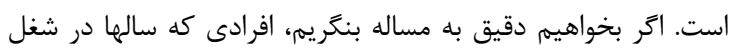
خود دوام مى آورند و آن را با علاقه دنبال مى كنند، در بـ حاليكه انتظارات

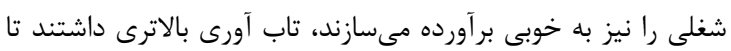

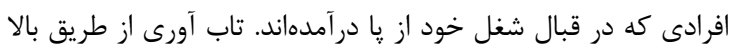

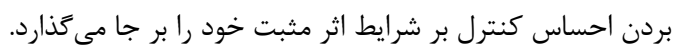

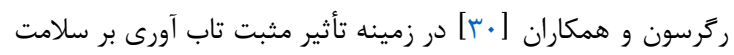

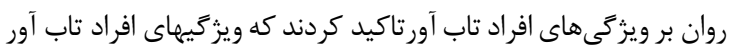

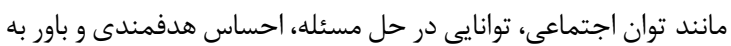

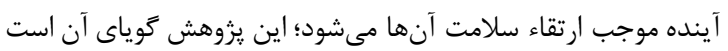

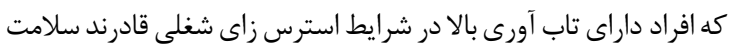

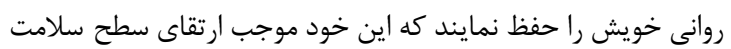

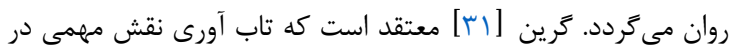

4. Maxwell A, Riley P. Emotional demands, emotional labour and occupational outcomes in school principals. Educ Manage Adm Leadersh 2016;45(3):484-502. doi: 10.1177/1741143215607878

5. Drach-Zahavy A, Yagil D, Cohen I. Social model of emotional labour and client satisfaction: exploring interand intrapersonal characteristics of the client-provider encounter. Work Stress. 2017;31(2):182-208. doi: $10.1080 / 02678373.2017 .1303550$

6. Grandey A. Emotion regulation in the workplace: A new way to conceptualize emotional labor. J Occup Health Psychol. 2005;1:95-110.

7. Gosserand RH, Diefendorff JM. Emotional display rules and emotional labor: the moderating role of commitment. J Appl Psychol. 2005;90(6):1256-64. doi: 10.1037/00219010.90.6.1256 pmid: 16316278 
8. Bondarenko Y, du Preez E, Shepherd D. Emotional labour in mental health field workers. New Zealand J Psychol 2017;46(1):4.

9. Morris JA, Feldman DC. Managing emotions in the workplace. J Manag Issues. 1997:257-74.

10. Kidd S, Kenny A, McKinstry C. The meaning of recovery in a regional mental health service: an action research study. J Adv Nurs. 2015;71(1):181-92. doi: 10.1111/jan.12472 pmid: 25040239

11. Lereya ST, Copeland WE, Costello EJ, Wolke D. Adult mental health consequences of peer bullying and maltreatment in childhood: two cohorts in two countries. Lancet Psychiatry. 2015;2(6):524-31. doi: 10.1016/S2215-0366(15)00165-0 pmid: 26360448

12. Graham S. Attribution theory: Applications to achievement, mental health, and interpersonal conflict. Psychol Press. 2014;4:41-2.

13. Sharma P, Davey A, Davey S, Shukla A, Shrivastava K, Bansal R. Occupational stress among staff nurses: Controlling the risk to health. Indian J Occup Environ Med. 2014;18(2):52-6. doi: 10.4103/0019-5278.146890 pmid: 25568598

14. Johnston D, Bell C, Jones M, Farquharson B, Allan J, Schofield P, et al. Stressors, Appraisal of Stressors, Experienced Stress and Cardiac Response: A Real-Time, Real-Life Investigation of Work Stress in Nurses. Ann Behav Med. 2016;50(2):187-97. doi: 10.1007/s12160015-9746-8 pmid: 26608281

15. Robertson IT, Cooper CL, Sarkar M, Curran T. Resilience training in the workplace from 2003 to 2014: A systematic review. J Occup Organ Psychol 2015;88(3):533-62. doi: 10.1111/joop. 12120

16. Rees CS, Breen LJ, Cusack L, Hegney D. Understanding individual resilience in the workplace: the international collaboration of workforce resilience model. Front Psychol. 2015;6:73. doi: 10.3389/fpsyg.2015.00073 pmid: 25698999

17. Son Y-J, Gong H-H, You M-A, Kong S-S. Relationships between workplace violence experience and posttraumatic stress symptoms, resilience in clinical nurses. J Kor Data Anal Soci 2015;17(1):515-30.

18. Hsieh HF, Hung YT, Wang HH, Ma SC, Chang SC. Factors of Resilience in Emergency Department Nurses Who Have Experienced Workplace Violence in Taiwan. J Nurs Scholarsh. 2016;48(1):23-30. doi: 10.1111/jnu. 12177 pmid: 26580490

19. Zapf D, Vogt C, Seifert C, Mertini H, Isic A. Emotion Work as a Source of Stress: The Concept and Development of an Instrument. Eur J Work Organ Psychol 1999;8(3):371-400. doi: $10.1080 / 135943299398230$

20. Montazeri A, Harirchi AM, Shariati M, Garmaroudi G, Ebadi M, Fateh A. The 12-item General Health
Questionnaire (GHQ-12): translation and validation study of the Iranian version. Health Qual Life Outcomes. 2003;1:66. doi: 10.1186/1477-7525-1-66 pmid: 14614778

21. Connor KM, Davidson JR. Development of a new resilience scale: the Connor-Davidson Resilience Scale (CD-RISC). Depress Anxiety. 2003;18(2):76-82. doi: 10.1002/da. 10113 pmid: 12964174

22. Mohammadi M. Effective factors on resiliency in individuals at risk for substance abuse. Tehran, Iran: University of Welfare and Rehabilitation; 2006.

23. Cho Y-N, Rutherford BN, Park J. Emotional labor's impact in a retail environment. J Busin Res 2013;66(11):2338-45. 10.1016/j.jbusres.2012.04.015

24. Friborg O, Hjemdal O, Rosenvinge JH, Martinussen $\mathrm{M}$, Aslaksen PM, Flaten MA. Resilience as a moderator of pain and stress. J Psychosom Res. 2006;61(2):213-9. doi: 10.1016/j.jpsychores.2005.12.007 pmid: 16880024

25. Grandey AA, Fisk GM, Mattila AS, Jansen KJ, Sideman LA. Is "service with a smile" enough? Authenticity of positive displays during service encounters. Organ Behav Hum Decision Proc 2005;96(1):38-55. doi: 10.1016/j.obhdp.2004.08.002

26. Kim HJ. Hotel service providers' emotional labor: The antecedents and effects on burnout. Int J Hosp Manage 2008;27(2):151-61. doi: 10.1016/j.ijhm.2007.07.019

27. Pisaniello SL, Winefield HR, Delfabbro PH. The influence of emotional labour and emotional work on the occupational health and wellbeing of South Australian hospital nurses. J Vocat Behav. 2012;80(3):579-91. doi: 10.1016/j.jvb.2012.01.015

28. Shichiri K, Shibuya M, Murayama K, Sato C, Kaminushi $\mathrm{K}$, Uenoyama T, et al. Features of Developmental Level of Defense Mechanisms and Adjustment Status of University Students in Japan. Health. 2015;07(01):52-7. doi: 10.4236/health.2015.71007

29. Karimi L, Leggat SG, Donohue L, Farrell G, Couper GE. Emotional rescue: the role of emotional intelligence and emotional labour on well-being and job-stress among community nurses. J Adv Nurs. 2014;70(1):176-86. doi: 10.1111/jan. 12185 pmid: 23763612

30. Rogerson S, Meir R, Crowley-McHattan Z, McEwen K, Pastoors R. A Randomized Controlled Pilot Trial Investigating the Impact of a Workplace Resilience Program During a Time of Significant Organizational Change. J Occup Environ Med. 2016;58(4):329-34. doi: 10.1097/JOM.0000000000000677 pmid: 27058471

31. Green JP, Wallace DM, Hargrove AK. Exploring Workplace Resilience Through a Personality Strength Lens. Ind Organ Psychol 2016;9(02):447-52. doi: 10.1017/iop.2016.37 